\title{
Report of the Annual Meeting
}

Rapports annuels de la Société historique du Canada

\section{La fondation du Séminaire de Québec}

\section{Honorius Provost}

Volume 42, numéro 1, 1963

URI : https://id.erudit.org/iderudit/300611ar

DOI : https://doi.org/10.7202/300611ar

Aller au sommaire du numéro

Éditeur(s)

The Canadian Historical Association/La Société historique du Canada

ISSN

0317-0594 (imprimé)

1712-9095 (numérique)

Découvrir la revue

Citer cet article

Provost, H. (1963). La fondation du Séminaire de Québec. Report of the Annual Meeting / Rapports annuels de la Société historique du Canada, 42(1), 19-30. https://doi.org/10.7202/300611ar d'utilisation que vous pouvez consulter en ligne. 


\title{
LA FONDATION DU SEMINAIRE DE QUEBEC
}

\author{
Honorius Provost \\ Archives du Séminaire de Québec
}

Dans les bulles que l'évêque élu de Québec, après certaines difficultés, réussit enfin à obtenir, comme titulaire du siège de Pétrée in partibus infidelium et vicaire apostolique au Canada, bulles datées du premier juin 1658, il n'est fait aucune allusion au recrutement du clergé ni à la fondation d'un séminaire à cette fin. Dans les facultés et privilèges concédés peu après par le Pape Alexandre VII au futur prélat, rien non plus qui recommande une attention particulière à la question; seulement le privilège bien ordinaire, si la nécessité et la rareté des prêtres se font sentir, d'anticiper d'un an l'âge canonique du sacerdoce et de passer outre aux temps et aux intervalles prescrits pour la collation des ordres.

Sur le point de partir pour le Canada, Mgr de Laval choisit pour compagnons trois ecclésiastiques, disciples comme lui-même de Jean de Bernières de Louvigny: deux prêtres, les abbés Philippe Pèlerin et Jean Torcapel, et un clerc tonsuré, Henri de Bernières, neveu du directeur de l'ermitage de Caen. Ce maitre en spiritualité, qui devait quitter le monde dès le 8 mai 1659, rédigea à leur intention, en forme de testament spirituel, des règles de conduite empreintes du plus haut mysticisme. ${ }^{1}$ On y trouve quelques conseils au nouveau vicaire apostolique, pour la gouverne de son église, mais rien d'explicite en ce qui concerne la formation du clergé. Ce n'est donc pas dans les instructions reçues au départ, mais dans les actes et les événements subséquents qu'il nous faut chercher les présages et l'esprit de la fondation d'un séminaire, à laquelle nous allons bientôt assister.

Partis de France le jour de Pâques, ${ }^{2} 13$ avril 1659, Mgr de Laval et ses compagnons débarquèrent à Québec le 16 juin suivant. N'y ayant point de presbytère ni de résidence disponible pour recevoir l'évêque, on l'hébergea d'abord chez les Jésuites, puis à l'Hôtel-Dieu, dans une chambre des pauvres. Mais, désireux de ne pas être à charge et surtout de retenir près de soi sa petite famille spirituelle, pour reprendre à Québec la vie de l'ermitage de Caën, il loua des Ursulines une modeste maison près de leur monastère, dite maison de Mme de la Peltrie, où il demeura plus de deux ans. Le 6 novembre 1661, il retournait cependant, avec tout son monde, habiter des appartements plus amples et plus commodes, chez les Jésuites. "Ses gens, dit le Journal des

1 Abbé Bertrand de La Tour, Mémoires sur la vie de M. de Laval, premier évêque de Québec, Cologne, 1761, pp. 25-30.

2 De La Tour, op. cit., p. 21. 
Jésuites, prenaient leurs repas avec les pensionnaires". Il doit s'agir là des serviteurs et tout au plus d'un ou deux séminaristes.

Le personnel ecclésiastique subit vite des changements. D'abord, les deux prêtres Pèlerin et Torcapel, après le premier hivernement, ne se sentirent point de taille à supporter davantage notre climat. Ils repassèrent en France l'automne suivant, pour être remplacés par l'abbé Thomas Morel, en 1661, et l'abbé Jean Dudouyt, en 1662. Entre temps, le séminariste Henri de Bernières reçut les ordres mineurs, le 2 décembre 1659, et la prètrise, le 13 mars 1660. Puis un autre séminariste, natif du pays, celui-là, Germain Morin, reçut la tonsure et les ordres mineurs en même temps que $M$. de Bernières; ordonné finalement en 1665, il devait être le premier prêtre canadien. Un troisième séminariste, Louis Jolliet, qui ne devait pas toutefois persévérer, se joignit à la famille en 1662. Ce fut, avant la lettre, l'existence et la vie commune d'un séminaire, un embryon de séminaire, mais déjà suffisant pour laisser pressentir les intentions et les réalisations futures de $\mathrm{Mgr}$ de Laval. Déjà, il avait réparti les tâches apostoliques au pays, attribuant aux Jésuites les missions chez les indiens, et aux prêtres séculiers la desserte des colons dispersés sur les côtes. Mais ce clergé diocésain, à part les Sulpiciens de Montréal, il lui incombait personnellement d'en assurer le recrutement.

L'état précaire de la colonie, harcelée et décimée par les Iroquois, presque totalement abandonnée par la Compagnie de la NouvelleFrance, troublée en son sein même par la traite des boissons enivrantes avec les tribus sauvages alliées des Français, avait quelque chose de lamentable, depuis quelques années, et on ne parlait rien moins que d'abandonner la partie pour rapatrier toute la colonie dans la vieille France. Mais Mgr de Laval se dressa énergiquement contre cette suggestion et décida en 1662, sans attendre la fermeture de la navigation saisonnière, de partir lui-même pour la mère-patrie, dans le but d'ouvrir les yeux des puissances sur les besoins urgents du Canada. Il s'embarqua donc à Québec, dès le 12 du mois d'août.

Le besoin de nouveaux prêtres, certes, devait être au nombre des desiderata. Mais allait-il déjà jusqu'à poser dans l'esprit du prélat l'idée précise d'un séminaire à fonder. On peut toujours le supposer, comme le fait l'historien Gosselin.3 "Il voyait la nécessité d'augmenter son clergé, écrit-il, de lui assurer un revenu et de mettre cette Eglise naissante en état de se suffire à elle-même. L'esprit des fondateurs du séminaire des Missions Étrangères, dont il était lui-même si rempli, a toujours été de travailler à la formation d'un clergé indigène. Il voulait donc établir un séminaire, et aussi un chapitre. Pour cela, il avait besoin, suivant l'usage et le droit de l'époque, de l'autorisation et de la sanction

3 Auguste Gosselin, Vie de Mgr de Laval, vol. I, p. 323. 
du roi." Soyons un peu moins péremptoire que le brave abbé Gosselin. S'il est permis de croire que Mgr de Laval songeait à quelque chose de tel, rien du moins n'en avait transpiré dans les documents qui nous sont parvenus, surtout dans ses lettres au Saint-Siège, où il se montre pourtant assez expansif.

On entendait, dans ce temps-là, sous le nom de séminaire, des institutions plus variées que celles d'aujourd'hui. Le droit canonique a réduit beaucoup l'extension de ce terme. Outre les séminaires pour la formation des clercs, demandés par le Concile de Trente, on avait, au 17ième siècle, des maisons de formation religieuse pour la jeunesse, des pensionnats surtout, qu'on appelait "séminaires" au moins par analogie. Marie de l'Incarnation parle dans ses lettres, du séminaire tenu par les Ursulines de Québec pour les petites sauvagesses, tant paiennes que baptisées. Et, quand les Relations des Jésuites font allusion à leur séminaire, il s'agit de leur collège de Québec ou du moins de leur groupe de pensionnaires.

Étant donné l'existence de ce collège secondaire établi depuis assez longtemps à Québec, Mgr de Laval ne pouvait songer dès l'abord qu'à une maison de formation du clergé dans sa dernière étape, le grand séminaire. C'était déjà plus que le nombre des candidats au sacerdoce ne pouvait exiger avant bien longtemps et l'on était en mesure de satisfaire cette exigence sans avoir recours si tôt à un séminaire en forme. Le recrutement nécessaire du clergé, il faudrait l'attendre de la vieille France plus que de la nouvelle.

Mais, précisément, conme le fait remarquer l'historien de La Tour, ${ }^{4}$ "les Ecclésiastiques que le zèle attirait dans ces pays lointains, et dont le plus grand nombre était pauvre, avaient à craindre de manquer du nécessaire, surtout dans un âge avancé et un état d'infirmité . . . Ces inconvénients arrêtaient ou faisaient périr grand nombre de bons ouvriers. M. de Laval était trop bon et trop équitable pour n'en être pas touché et s'efforcer d'y remédier. Il voulut que tout le Clergé ne fît qu'une famille, que la maison de l'Évêque fût la maison commune de tous les Ecclésiastiques, et le centre de tout le temporel, comme du spirituel; il s'engagea de fournir à tous tout ce qui leur serait nécessaire. Il voulut que quand les affaires, les maladies, la piété, les attireraient à Québec, ils trouvassent chez lui un asile toujours ouvert, qu'ils y vinssent même chaque année faire une retraite, et que quand l'âge ou les infirmités les mettraient hors d'état de servir, ils y eussent une ressource assurée, la nourriture et l'entretien jusqu'à la fin de leurs jours, et des prières après leur mort. Par ce moyen les Missionnaires, déchargés de toute sollicitude temporelle, se livraient au travail avec plaisir et sans réserve. Il exécuta ce pieux dessein jusqu'à l'établissement de son Séminaire. Dès qu'il l'êtit bâti, il lui donna tous ses biens, s'y

4 De La Tour, Mémoires..., p. 35. 
consacra, s'y renferma lui-même, et le chargea de maintenir cette union, et de remplir et perpétuer ses vues; ce qui subsista jusqu'à ce qu'un nouveau gouvernement changea la face des choses" (Allusion à la réforme de $\mathrm{Mgr}$ de Saint-Vallier).

Mais n'anticipons pas. Cette citation du premier biographe de Mgr de Laval a voulu donner en bref la genèse de l'établissement du Séminaire de Québec. Les documents officiels justifient l'assertion de point en point. Jusqu'ici, Mgr de Laval n'a rien dit de son projet de séminaire. Mais voici le moment où il prend la parole. Il est à Paris, à la fin de cet hivernement de 1662-63, qui aura été si précieux pour la survivance de la colonie française au Canada. Et c'est là que, le 26 mars 1663, il rédige son mandement d'érection du Séminaire de Québec. ${ }^{5}$

"François, par la grâce de Dieu et du Saint Siège, Evêque de Pétrée, Vicaire Apostolique en Canada, dit la Nouvelle France, nommé par le Roi premier évêque du dit pays, lorsqu'il aura plu a Notre Saint Père le Pape y ériger un évêché, à tous ceux qui ces présentes lettres verront salut en Notre Seigneur ..." Passons aux points saillants du document: "Considérant . . . qu'il est d'une extrême importance, dans ces commencements, de donner au Clergé la meilleure forme qui se pourra, pour perfectionner des ouvriers et les rendre capables à cultiver cette nouvelle vigne du Seigneur, en vertu de l'autorité qui Nous a été commise, Nous avons érigé et érigeons dès à présent et à perpétuité, un séminaire pour servir de Clergé à cette nouvelle Eglise, . . . dans lequel l'on élèvera et formera les jeunes clercs qui paraitront propres au service de Dieu, ... . et en outre, afin que l'on puisse dans le dit Séminaire et Clergé former un Chapitre, qui soit composé d'Ecclésiastiques du dit Séminaire, ... nous désirons que ce soit une continuelle école de vertu et un lieu de réserve, d'où nous puissions tirer des sujets pieux et capables, pour les envoyer à toutes rencontres et au besoin dans les paroisses et tous autres lieux du dit pays, afin d'y faire les fonctions curiales et autres auxquelles ils auront été destinés, et les retirer des mêmes paroisses et fonctions, quand on le jugera à propos ... Et d'autant qu'il est absolument nécessaire de pourvoir le dit Séminaire et Clergé d'un revenu capable de soutenir les charges et les dépenses qu'il sera obligé de faire, Nous lui avons appliqué et appliquons, affecté et affectons, dès à présent et pour toujours, toutes les dîmes de quelque nature qu'elles soient, . . . pour être possédées en commun et administrées par le dit Séminaire suivant nos ordres, .. . à condition qu'il fournira la subsistance de tous les Ecclésiastiques qui seront délégués dans les paroisses et autres endroits du dit pays, . . . qu'il entretiendra tous les dits ouvriers évangéliques, tant en santé qu'en maladie, soit dans leurs fonctions, soit dans la communauté, lorsqu'ils y seront rappelés, qu'il fera les frais de leurs voyages, quand on les tirera de IX, no 1 .

5 Archives du Séminaire de Québec (désormais ASQ), carton Polygraphie, 
France ou qu'ils y retourneront, ... . et, comme il est nécessaire de bâtir plusieurs églises,... Nous ordonnons (sans préjudice néanmoins de l'obligation que les peuples de chaque paroisse ont de fournir à la bâtisse des dites églises) qu'après que le dit Séminaire aura fourni toutes les dépenses annuelles, ce qui pourra rester de son revenu sera employé à la construction des églises, en aumônes et en autres bonnes oeuvres pour la gloire de Dieu et pour l'utilité de l'Église, selon les ordres de l'Évêque, sans que toutefois Nous, ni les successeurs évêques du dit pays, en puissions jamais appliquer quoi que ce soit à nos usages particuliers, nous ôtant même et aux dits Évêques la faculté de pouvoir aliéner aucun fonds du dit Séminaire, en cas de nécessité, sans l'exprès consentement de quatre personnes du corps du dit Séminaire et Clergé, savoir le supérieur, les deux assistants et le procureur."

La lourdeur de ce texte, dans la forme juridique et le style dixseptième siècle, ne le rend pas inintelligible. Au contraire, une idée maitresse s'inscrit dès le début et domine ensuite tout au long. L'établissement que fonde Mgr de Laval, c'est "un Séminaire pour servir de Clergé" à son vicariat apostolique. Les mots "Séminaire et Clergé" reviennent ensuite juxtaposés et comme synonymes à chaque fois. I1 n'y a pas à s'y méprendre: le Séminaire que veut le fondateur, c'est la communauté de son clergé séculier ou encore un diocèse communautaire. Il n'en parle pas si explicitement encore, mais on le sait par ailleurs, et le régime sera en vigueur durant tout son épiscopat, sinon davantage. Cette institution se fondait sur la désappropriation, c'est-à-dire sur la mise en commun de tous les revenus de chacun de ses membres. La préparation des clercs au sacerdoce intervenait dans ce cadre, mais seulement comme une fonction et un moyen de réaliser le plan général.

N'est-ce pas un écho fidèle de cette conception que l'on retrouve dans un ancien règlement de la maison, datant d'avant 1713, pour ne citer que celui-là ?" "Ce Séminaire a été institué principalement pour avancer le royaume de Dieu dans l'établissement et le progrès de la religion, soit parmi les Français de la colonie, soit parmi les Sauvages et infidèles du Canada; ce sont là les deux objets qu'on se doit proposer dans ce Séminaire. C'est donc l'établissement solide de la religion chrétienne parmi les nations privées de la foi et sa conservation parmi les Français de la colonie qui sont la principale fin de cette maison. Cette fin principale engage les directeurs qui seront chargés de la conduite de la maison à formet sur ce pied-là les jeunes clercs qui seront confiés à leur soin, de sorte qu'on en puisse tirer des Missionnaires pour les infidèles aussi bien que pour les Français . . ." Il y a dans ce texte un anachronisme révélateur, preuve qu'on a reproduit un texte plus ancien; car, au moment de sa rédaction, ce qui était vrai pour les missionnaires entretenus par le Séminaire chez les indiens de

6 ASQ, carton Séminaire 95, no 14, p. 1. 
l'Acadie et du Mississipi ne l'était plus pour les curés des paroisses canadiennes. A ce moment-là les cures avaient été fixées, les nominations relevaient de l'Évêque seul, les dîmes se percevaient à la façon normale et, pour toute fin pratique, les curés na'vaient plus de relations avec le Séminaire de Québec, sinon à titre d'anciens élèves toujours sympathiques à leur Alma Mater.

Mais revenons à $\mathrm{Mgr}$ de Laval. Sa fondation du Séminaire de Québec coincide tellement avec celle du Séminaire des Missions Étrangères de Paris que les historiens considèrent facilement le premier comme une filiale ou une succursale de celui-ci. Cependant il n'en fut pas ainsi dès le début et le mandement d'érection que nous avons analysé ne laisse pas pressentir dans son texte une intention dans ce sens. Bien au contraire, les objectifs paraissent notablement divergents et la constitution du Séminaire de Québec lui donne, sous l'autorité de l'Évêque, une parfaite autonomie. Il faudra trouver ailleurs le mobile de l'union réalisée entre les deux institutions par acte du 29 janvier 1665 .

Contrairement au Séminaire de Paris, qui avait déjà l'existence et une vie organique avant d'être muni de la reconnaissance officielle, le Séminaire de Québec eut son acte de fondation et ses lettres patentes royales dès le début, avant que d'être constitué pour de bon. A ne regarder que les documents, le Séminaire de Québec pourrait même se dire l'ainé de quelques mois de celui de Paris. Il pouvait en être autrement dans les intentions. Mais, comme Mgr de Laval, de passage en France pour un hiver seulement, en 1663 , ne pouvait attendre la fin des ennuyeuses procédures qui compliquaient la fondation du Séminaire de Paris, il brûla en quelque sorte les étapes, en réalisant séparément sa fondation, tant au for canonique qu'au for civil, remettant à plus tard de l'y rattacher, comme un greffon sur une tige plus robuste. Du moins, c'est l'interprétation que nous croyons justifiable de donner aux pièces documentaires qui nous sont parvenues.

Parmi celles-ci, il en est une fort intéressante que nous avons retenue, qui semble avoir échappé aux biographes de $\mathrm{Mgr}$ de Laval, même à l'abbé Auguste Gosselin, peut-être parce qu'ils n'ont pu l'identifier. C'est un petit document de deux pages et un quart d'écriture, ni signé ni daté, mais incontestablement de la main de Mgr de Laval et de la main la plus appliquée que nous lui connaissions. C'est lui, d'ailleurs, qui 1'a endossée de ces mots: "Raisons qui concernent l'établissement d'un séminaire en la nouvelle France"; puis une autre main a ajouté: "et attachent les dixmes aud[it] Seminaire". Quant à la date, c'est le texte même qui nous permet de la fixer après le 26 mars 1663, date du mandement d'érection, mais avant les lettres patentes de Louis XIV, qui sont du mois d'avril suivant. Mgr de Laval avait, à ce moment-là, toutes les bonnes grâces de Louis XIV, qui 
l'appelait complaisamment son cousin, parce qu'ils étaient tous deux descendants de la famille des Montmorency. L'évêque, désireux d'obtenir la reconnaissance légale pour sa nouvelle fondation, a dû préparer ce mémoire à l'intention du ministre ou du procureur du Roi chargé d'octroyer les lettres patentes. Peut-être n'eut-il pas à l'utiliser, mais qu'importe. Plus que tout autre ce document donne le fond de la pensée du fondateur, ou du moins toutes les raisons valables officiellement qu'il avait pour instituer son Séminaire dans la forme que nous avons vue. ${ }^{7}$ "Tous les évêques, écrit-il, sont obligés d'établir des séminaires, pour maintenir tous les ecclésiastiques dans un état de vie qui soit convenable à leur condition. Il est nécessaire en la NouvelleFrance plus qu'en aucun autre lieu d'y en avoir un, $1^{\circ}$ parce que l'on ne peut trouver d'ecclésiastiques en France qui veuillent $y$ aller et quitter leur établissement à moins qu'il ne soient assurés d'y passer leur vie dans un lieu où l'on se charge d'eux; $2^{\circ}$ leurs fonctions sont si continuelles qu'elles accablent les prêtres qui y sont employés, que souvent et pendant un temps considérable à peine peuvent-ils avoir le moyen de dire leur office, et à moins que de se renouveler dans un séminaire, ils se dégoûtent et repassent en France, ce qui m'est arrivé en plusieurs bons ecclésiastiques.

Ce qui m'a obligé d'attribuer toutes les dixmes au Séminaire aux conditions portées par l'établissement, est:

$1^{\circ}$ les dixmes appartiennent de droit divin à l'évêque et aux prêtres qui administrent les paroisses;

$2^{\circ}$ cela étant supposé, il y a tout sujet d'appréhender que les évêques qui me succèderont ne prennent la meilleure part de ces dixmes pour eux et qu'ils n'en laissent la moindre aux prêtres qui desserviront les paroisses, comme dans la plupart des diocèses de France, où des prêtres n'ont qu'une pension fort modique, ce qui a bien de mauvais effets... $6^{\circ}$ quoique, dans 1'établissement, il soit dit que le Séminaire jouira des dixmes et que la chose soit pour le présent, cependant, pendant que je vivrai, j'espère bien ne pas appliquer ce fonds à l'entretien du dit Séminaire, mais continuer la construction des églises, qui par ce moyen ne coûte rien aux habitants, qui est néanmoins tellement nécessaire, qu'il est dès à présent comme impossible d'avoir soin de leurs âmes, à moins que d'y pourvoir au plus tôt. Il y a trente ans à peu près que les Trois-Rivières et le Montréal sont établis et il n'y a pas encore d'église à l'un ni à l'autre, ce qui cause des maux qui sont grands et auxquels l'on ne saurait apporter de remède.

$7^{\circ} \mathrm{Je}$ trouve les lettres patentes du Roi nécessaires, parce que, bien qu'elles ne le soient pas pour l'établissement des autres séminaires de France, par l'avis des avocats, néamoins elles doivent beaucoup affermir ce dépouillement que l'évêque fait du droit qu'il a aux dixmes, afin

7 ASQ, carton Séminaire 2, no 27. 
que les évêques qui nous succéderont ne jugent pas que l'évêque seul les ait liés."

C'est entièrement dans ces intentions et selon cette formule, que Mgr de Laval reçut du Roi ses lettres patentes, signées LOUIS et datées simplement "d'avril, l'an de grâce mil six cent soixante-trois"; car les rois de France, dans leurs actes solennels, n'avaient pas l'habitude d'inscrire le quantième du mois. Le corps du document ${ }^{8}$ reproduit presque textuellement le mandement d'érection et, qui plus est, la calligraphie est absolument semblable, sauf pourl es deux dernières lignes. Ceci laisse à supposer que Mgr de Laval, recourant à l'un des nombreux scribes anonymes qui peuplaient les chancelleries, à dirigé lui-même la rédaction des lettres patentes royales. C'était, d'ailleurs, le moyen le plus sûr d'obtenir la teneur qu'il désirait. Il avait déjà éprouvé pour son compte quels mauvais tours l'on pouvait se faire jouer par les intrigues de cour, lors de l'émission de son brevet royal comme vicaire apostolique du Canada, le 27 mars 1659.

Pour cette fois, cependant, il parait n'y avoir eu aucune intervention fâcheuse, puisque toute l'affaire était réglée en un temps record. Ayant réussi toutes ses démarches à la Cour, au-delà même de ce qu'il espérait, Mgr de Laval se rembarquait pour le Canada, au milieu de mai, et, après une traversée de près de quatre mois, marquée par les tempêtes et la maladie des équipages, il mettait pied à terre à Québec, le 15 septembre 1663, avec, entre autres compagnons, l'abbé Louis Ango de Maizerets, qui devait être, selon l'expression de l'abbé Auguste Gosselin, "un des piliers de son Séminaire et de son Église".

Mgr de Laval rendit compte en général au Saint-Siège de son fructueux voyage en France, dans ses lettres du 24 et du 25 octobre 1663. Il envoya pareillement, en 1664, un long rapport de Statu Ecclesiae. Et c'est alors qu'il parle pour la première fois de sa fondation. ${ }^{9}$ Le texte est en latin et nous le traduisons: "J'ai établi, dit-il, dans cette Église du Canada, un séminaire, où l'on forme à la discipline ecclésiastique les jeunes Canadiens qui doivent être promus au sacerdoce . . . J'ai réussi, à force de travail et d'industrie, à assurer à ce séminaire un revenu suffisant pour le faire subsister, en y ajoutant celui des paroisses, que j'ai toutes unies au séminaire. Le roi a tout confirmé de son autorité souveraine" . . . Plus loin, il ajoute: "J'ai établi mon domicile dans mon séminaire. Il y a là avec moi huit prêtres, que j'envoie, suivant les besoins et à ma discrétion, dans les différentes missions de mon vicariat, ou que j'occupe sans relâche à d'autres fonctions ecclésiastiques". A l'automne de 1665, alors qu'il a déjà

8 ASQ, carton Séminaire 11 , no 1.

9 Mgr de Laval désirait en avoir une approbation écrite du Saint-Siège et c'est alors qu'il a dû préparer, pour la faire parvenir à Rome, une version latine de l'acte d'érection (ASQ, carton Séminaire 1, no 8). 
uni son Séminaire à celui des Missions Étrangères de Paris, il déclare au Saint-Siège le fait et le plan de sa fondation. Retenons seulement, des trois documents où il en fait mention, cet extrait, traduit de la lettre du 24 octobre, au Pape Alexandre VII :10 "Pour poser un premier fondement de vraie piété et de religion dans ces contrées nouvellement colonisées, j'ai cru qu'un Séminaire de Prêtres était tout à fait indiqué et même indispensable. Alors j'ai commencé à l'organiser et, dès ce commencement, je l'ai agrégé au Séminaire de Paris pour les Missions étrangères, qui a déjà reçu son approbation de l'Eminentissime Cardinal Légat. Et j'ai cru très utile d'attacher à mon Séminaire la paroisse de Québec. Non pas qu'elle lui fournisse à présent le secours temporel qui serait nécessaire; mais c'est afin qu'on puisse y former ensemble aux fonctions ecclésiastiques qu'ils rempliront un jour les adolescents de ce pays qui en auront les aptitudes. Car, il n'est pas trop facile d'obtenir de France des ouvriers adaptés aux travaux de l'Église canadienne... Entre temps, par le moyen des prêtres séculiers de notre Séminaire, nous dirigeons autant que nous pouvons, un peu de tous côtés, la vie chrétienne. C'est ainsi que l'un d'eux dessert des familles dispersées sur une distance de six lieues". Dans ce texte, on aura encore remarqué l'expression de l'auteur: "Séminaire de Prêtres"; d'ailleurs, les attributions qu'il confie à se Séminaire sont celles qui conviennent à un clergé diocésain en général.

Dans une lettre de Mgr de Laval, quelques années après, précisément du 8 novembre 1668, le vicaire apostolique raconte familièrement à son grand ami, l'abbé Poitevin, curé de Saint-Josse, à Paris, les événements récents du Canada. L'original de cette lettre est disparu; mais les Jésuites avaient eu la bonne inspiration de l'inclure dans leur Relation de 1667-68. A ce moment-là, Mgr de Laval venait d'ouvrir le Petit Séminaire de Québec. Il écrit, à ce sujet, vers la fin de sa lettres:11 "Les Prêtres de notre Séminaire des Missions Étrangères, ne nous ayant pas moins fait paraitre de soin et de vigilance dans l'éducation des enfants de ce pays, que nous leur avons donnés à former à l'état ecclésiastique, qu'ils nous ont donné des marques de leur zèle dans les travaux qu'il y a à souffrir dans tous les lieux des habitations de ce pays où nous les employons; Nous avons estimé ne pouvoir rien faire qui soit plus à la gloire de Dieu et pour le bien de notre Église, que de leur confier de nouveau la direction de ce second Séminaire: d'autant plus que nous avons jugé à propos de le renfermer dans l'enceinte de notre Séminaire dans laquelle nous avons fait accomoder un logement propre à ce dessein."

Que voit-on, dans ce document, sinon une extension et une attribution nouvelle dans l'oeuvre du Séminaire de Québec? Celui-ci reste

10 Nova positio super virtutibus, pp. 258-259.

11 Thwaites, The Jesuit Relations, vol. 52, pp. 48-50. 
ce qu'il était, depuis le début, et ce qu'il restera, dans l'esprit de son fondateur, tout le long de l'épiscopat de Mgr de Laval: la communauté du clergé diocésain. D'ailleurs, d'autres faits sont là, et même des chiffres, pour corroborer. On les trouve consignés dans les livres de comptes du Séminaire. Les dixmes étant insuffisantes et souvent n'étant pas exigées, l'Évêque avait obtenu du Roi un supplément annuel de quelques milliers de francs, pour aider le Séminaire à supporter ses charges d'entretien du clergé et de construction des églises. L'Évêque y mettait du sien aussi et présidait à la répartition des subsides, selon les besoins, par le Séminaire. On trouve, dans les comptes, des entrées pour des églises aussi éloignées que celles de Sorel et de Boucherville; on en voit pour l'église de Lauzon et les plus anciennes de 1'Ile d'Orléans: Sainte-Famille, Saint-Pierre, Saint-Laurent et Saint-Jean. Les deux premières ont été bâties presque entièrement aux frais du Séminaire. On peut en dire autant pour les églises de la Côte de Beaupré: L’Ange-Gardien, Château-Richer, et surtout Sainte-Anne; le Séminaire a contribué largement aux reconstructions de cette dernière, en 1676 , et en 1691. Et nous ne parlons pas des comptes particuliers de chaque curé dépendant du Séminaire.

D'où venait à Mgr de Laval cette conception inédite d'un séminaire et d'un clergé diocésain? Certainement pas du régime des diocèses de France. L'abbé Auguste Gosselin établit un rapprochement entre l'organisation susdite et celle du vénérable Barthélemy Holzhauser, fondateur des Barthélemites d'Allenagne, congrégation de prêtres séculiers vivant en commun. Cet institut datait de 1640, mais il est peu probable que Mgr de Laval en ait eu suffisamment connaissance pour entreprendre de le reproduire en Canada. Et si c'eût été le cas, il en aurait parlé quelque part. Ce que nous croyons plus probable, c'est qu'il a voulu reconstituer ici l'atmosphère, ainsi que la vie spirituelle et apostolique de la Société des Bons Amis et de l'Ermitage de Caen, avec des prêtres qui, d'ailleurs, s'y étaient initiés pour la plupart.

De plus, Mgr Laval voulait une organisation religieuse à la mesure de 1'Eglise qu'on lui avait confiée. Ce n'était pas un diocèse adulte, mais un diocèse à fonder. Ce qui nous paraîtrait aujourd'hui un cadre étouffant, une centralisation inconcevable, paraissait nécessaire, dans un pays neuf et pauvre, un vrai pays de mission. Mgr de Laval n'avait-il pas voulu un apostolat missionnaire? N'appelait-il pas ses embryons de paroisses des missions, et ses curés des missionnaires? Or, dans quel territoire de mission, encore de nos jours, voit-on des prêtres séculiers isolés, abandonnés à leurs propres ressources?

L'évêque de Pétrée n'avait rien fait, en tout cas, qu'avec l'approbation du Roi et du Saint-Siège. Et ce régime pouvait durer assez longtemps, pourvu que l'Évêque de Québec, quel qu'il fût, demeurât étroitement uni et en quelque sorte indentifié à son Séminaire. Mgr 
de Laval, qu'on prétend avoir été si autoritaire et despotique, a pourtant pratiqué toute sa vie ce régime d'effacement. Mais, s'il est vrai que de tels cadres devaient venir à céder fatalement, un jour, il eut la douleur de subir cette échéance plus tôt qu'il ne s'y attendait et d'assister, impuissant, à la démolition de son édifice à peine commencé.

Ce fut d'abord Louis XIV, qui, par une ordonnance sur les dixmes et la fixation des cures, en mai 1679 , détruisit en principe l'influence du Séminaire sur les paroisses, instituée par ses lettres patentes de 1663 . Puis, dès les premières années de son règne, $\mathrm{Mgr}$ de Saint-Vallier, voulut reconstruire le diocèse sur le modèle des diocèses de France et, pour cela, prit à ses charges l'ouverture des paroisses et la construction des églises, s'opposa à toute ingérence du Séminaire auprès des curés et le réduisit à la condition de simple séminaire diocésain. Ce fut une purification passive, très pénible pour le fondateur, qui sut cependant l'accepter de la volonté divine.

On attribue aujourd'hui le mérite de notre organisation paroissiale canadienne-française à Mgr de Saint-Vallier, ce qui n'est vrai qu'en partie; la chose était commencée avant lui. Mais c'est à Mgr de Laval surtout et, par conséquent, à l'organisation religieuse qu'il avait conçue, qu'on attribue la discipline, l'union et l'édification générale dans la primitive Église du Canada.

Témoin l'abbé de La Tour, historien de Mgr de Laval, qui écrit:12 "C'est à cette union étroite que la religion fut redevable des rapides progrès qu'elle fit en Canada, et le Clergé de la piété édifiante qui faisait son caractère."

Témoin le Père Claude Dablon, supérieur des Jésuites, au début de la Relation de 1672: "Nous en éprouvons les effets par la continuation des bénédictions dont Dieu favorise et les travaux de nos missionnaires et ceux de MM. les Ecclésiastiques de son Église, qui continuent avec un grand zèle et l'édification publique à procurer l'honneur de Dieu, et à travailler au parfait établissement des paroisses dans toute l'étendue de ce pays: ce qui ne sert pas peu au progrès que fait notre Foi, qui n'avait pas encore été portée si loin, ni publiée avec plus succès."

Témoin surtout Mgr de Saint-Vallier lui-même, qui, après son premier voyage en Canada, comme évêque élu de Quẻbec, en 1685-86, rend compte de ses constations. Ce qu'il dit alors contraste avec ce qu'il dira, quelque temps après, en essayant de retirer les volumes qu'il avait déjà mis en circulation, sous le titre de: Estat present de l'Eglise et de la colonie françoise dans la Nouvelle France, Par M. l'Evêque de Québec. ${ }^{13}$ Mais, malheureusement pour lui, les écrits restent et,

12 Mémoires..., p. 34.

13 A Paris, Chez Robert Pepie, 1688, pp. 27 et 28. 
comme conséquence, nous pouvons dire que l'auteur ou bien manquait beaucoup du don d'observation, ou bien plutôt jugeait seulement d'après des impressions très versatiles. Voici donc ce qu'il écrivait, en 1688: "En arrivant à Québec, je fus descendre au Séminaire des Missions étrangères, qui est dépendant de celui de Paris et qui a été jusqu'à présent le Séminaire Épiscopal de Canada .. . Les Directeurs qui le gouvernent sont en petit nombre, et s'ils avaient moins de grâce et d'activité qu'il n'en ont, il leur serait impossible de faire tout ce qu'ils font au dedans et au dehors de leur maison: le détachement dont ils font profession, la charité qui les unit, l'assiduité qu'ils ont au travail, et la régularité qu'ils s'efforcent d'inspirer à tous ceux qui sont sous leur conduite, m'ont donné une très sensible consolation: quelque ferveur que j'aie trouvé parmi eux, j'ai eu le plaisir de la voir redoubler dans leur Maison ... Il me sembla pour lors voir revivre dans l'Église de Canada quelque chose de cet esprit de détachement qui faisait une des principales beautés de l'Eglise naissante de Jérusalem, du temps des Apôtres". . . 